\title{
Radial index and Euler obstruction of a 1-form on a singular variety
}

\author{
W.Ebeling and S.M.Gusein-Zade *
}

\begin{abstract}
A notion of the radial index of an isolated singular point of a 1-form on a singular (real or complex) variety is discussed. For the differential of a function it is related to the Euler characteristic of the Milnor fibre of the function. A connection between the radial index and the local Euler obstruction of a 1-form is described. This gives an expression for the local Euler obstruction of the differential of a function in terms of Euler characteristics of some Milnor fibres.
\end{abstract}

\section{Introduction}

An isolated singular point (zero) of a vector field or of a 1-form on a smooth manifold (real or complex) has a natural integer invariant - the index. There are several generalizations of this notion: an index of a vector field on an isolated singularity [1, 4, an index of a vector field [7, 11, or of a 1-form [5] on an isolated complete intersection singularity, ....

Let $(X, 0) \subset\left(\mathbb{R}^{N}, 0\right)$ be the germ of a purely $n$-dimensional analytic variety and let $X=\bigcup V_{i}$ be a Whitney stratification of it. Let $v$ be a continuous vector field on a neighbourhood of the origin in $\mathbb{R}^{N}$ tangent to $X$. The last fact means that the vector field $v$ is tangent to each stratum $V_{i}$ of the Whitney stratification of $(X, 0)$. Suppose that $v(x) \neq 0$ for all points $x$ from a punctured neighbourhood of the origin in $X$. In [4] there was defined a notion of an index of the vector field $v$ on $X$ at the origin which we shall call the radial index. (For a germ $(X, 0)$ with an isolated singular point at the origin this notion was defined and discussed in 1, 4.)

The condition for a vector field to be tangent to a germ of a singular variety is a very restrictive one. In particular, holomorphic vector fields with isolated singularities do not always exist.

\footnotetext{
*Partially supported by the DFG-programme "Global methods in complex geometry", grants RFBR-01-01-00739, NSh-1972.2003.1. Keywords: singular varieties, 1-forms, singular points, index, Euler obstruction. AMS Math. Subject Classification: 14B05, 32S60, $58 \mathrm{~A} 10$.
} 
Example. Consider the surface $X$ in $\mathbb{C}^{3}$ given by the equation

$$
x y(x-y)(x+z y)=0 .
$$

It has singularities on the line $\{x=y=0\}$. It is a family of four lines in the $(x, y)$-plane with different cross ratios. Then any holomorphic vector field tangent to $X$ vanishes on the line $\{x=y=0\}$, because the translation along such a vector field has to preserve the cross ratio of the lines.

However, 1-forms with isolated singular points always exist. Therefore (and because of some other advantages: see the discussion in [5]) we prefer to discuss 1-forms instead of vector fields.

Suppose that $\omega$ is a continuous 1 -form defined in a neighbourhood of the origin in $\mathbb{R}^{N}$. A singular point of the 1 -form $\omega$ on $X$ is a singular point of its restriction to a stratum of the Whitney stratification of $X$. (One should consider all points of a zero-dimensional manifold as singular ones.) Suppose that the 1-form $\omega$ has an isolated singular point on $X$ at the origin. In this situation we define the notion of the radial index of the 1-form $\omega$ on $(X, 0)$ (or rather adapt one for vector fields from [4). We also define a similar concept for complex 1-forms on a germ of a complex analytic subvariety of $\left(\mathbb{C}^{N}, 0\right)$.

For a germ $f$ of an analytic function on the germ $(X, 0)$ of an analytic variety, there exists the notion of the Milnor fibration and of the Milnor fibre (or of the Milnor fibres in the real setting: the positive and the negative ones): see, e.g., 10, 12. If the function $f$ has an isolated critical point on $X$ at the origin (i.e. if its differential $d f$ has an isolated singular point there) we describe a connection between the radial index of the differential $d f$ and the Euler characteristic of the Milnor fibre of the germ $f$. In the described situation there is also an appropriately adapted notion of the local Euler obstruction of a 1-form on the germ of a complex analytic variety [3]. We describe a connection between the radial index and the local Euler obstruction. This gives an expression for the local Euler obstruction of (the differential of) a function in terms of Euler characteristics of some Milnor fibres.

\section{The radial index of a 1 -form}

First let $(X, 0) \subset\left(\mathbb{R}^{N}, 0\right)$ be the germ of a real analytic variety and let $\omega$ be a (continuous) 1-form on a neighbourhood of the origin in $\mathbb{R}^{N}$.

Definition: The 1-form $\omega$ is radial on $(X, 0)$ if, for an arbitrary nontrivial analytic $\operatorname{arc} \varphi:(\mathbb{R}, 0) \rightarrow(X, 0)$ on $(X, 0)$, the value of the 1 -form $\omega$ on the tangent vector $\dot{\varphi}(t)$ is positive for positive $t$ (small enough).

Radial 1-forms exist: e.g. the differential $d \phi$ for the germ of an analytic function $\phi:\left(\mathbb{R}^{n}, 0\right) \rightarrow(\mathbb{R}, 0)$ with a strict local minimum at the origin (one can take $\phi=r^{2}$ where $r$ is the distance from the origin in $\left.\mathbb{R}^{N}\right)$.

Let $X=\bigcup_{i=0}^{q} V_{i}$ be a Whitney stratification of the germ $(X, 0), V_{0}=\{0\}$. Let $\omega$ be an (arbitrary continuous) 1-form on a neighbourhood of the origin in 
$\mathbb{R}^{N}$ with an isolated singular point on $(X, 0)$ at the origin. Let $\varepsilon>0$ be small enough so that in the closed ball $B_{\varepsilon}$ of radius $\varepsilon$ centred at the origin in $\mathbb{R}^{N}$ the 1-form $\omega$ has no singular points on $X \backslash\{0\}$. It is easy to see that there exists a 1-form $\widetilde{\omega}$ on $\mathbb{R}^{N}$ such that:

1) The 1-form $\widetilde{\omega}$ coincides with the 1-form $\omega$ on a neighbourhood of the sphere $S_{\varepsilon}=\partial B_{\varepsilon}$.

2) The 1 -form $\widetilde{\omega}$ is radial on $(X, 0)$ at the origin.

3) In a neighbourhood of each singular point $x_{0} \in\left(X \cap B_{\varepsilon}\right) \backslash\{0\}, x_{0} \in V_{i}$, $\operatorname{dim} V_{i}=k$, the 1 -form $\widetilde{\omega}$ looks as follows. There exists a (local) analytic diffeomorphism $h:\left(\mathbb{R}^{N}, \mathbb{R}^{k}, 0\right) \rightarrow\left(\mathbb{R}^{N}, V_{i}, x_{0}\right)$ such that $h^{*} \widetilde{\omega}=\pi_{1}^{*} \widetilde{\omega}_{1}+$ $\pi_{2}^{*} \widetilde{\omega}_{2}$, where $\pi_{1}$ and $\pi_{2}$ are the natural projections $\pi_{1}: \mathbb{R}^{N} \rightarrow \mathbb{R}^{k}$ and $\pi_{2}: \mathbb{R}^{N} \rightarrow \mathbb{R}^{N-k}$ respectively, $\widetilde{\omega}_{1}$ is the germ of a 1 -form on $\left(\mathbb{R}^{k}, 0\right)$ with an isolated singular point at the origin, and $\widetilde{\omega}_{2}$ is a radial 1 -form on $\left(\mathbb{R}^{N-k}, 0\right)$.

Remark. One can demand that the 1-form $\widetilde{\omega}_{1}$ has a non-degenerate singular point (and therefore $\operatorname{ind}_{0} \widetilde{\omega}_{1}= \pm 1$ ), however, this is not necessary for the definition.

Definition: The radial index ind $_{X, 0} \omega$ of the 1 -form $\omega$ on the variety $X$ at the origin is the sum

$$
1+\sum_{i=1}^{q} \sum_{Q \in \operatorname{Sing} \tilde{\omega} \cap V_{i}} \operatorname{ind}_{Q} \widetilde{\omega}_{\mid V_{i}}
$$

where the sum is taken over all singular points of the 1-form $\widetilde{\omega}$ on $(X \backslash\{0\}) \cap B_{\varepsilon}$.

Remarks. 1. One can write the definition as

$$
\sum_{i=0}^{q} \sum_{Q \in \operatorname{Sing} \widetilde{\omega} \cap V_{i}} \operatorname{ind}_{Q} \widetilde{\omega}_{\mid V_{i}}
$$

(i.e. over all singular points of the 1-form $\widetilde{\omega}$ on $X \cap B_{\varepsilon}$ ) if one assumes that the index of a 1-form on a point (a zero-dimensional manifold) is equal to 1.

2. Since no other indices will be considered in this paper, we shall usually omit the word radial.

To prove that this notion is well defined, we shall show that not only the total sum

$$
\sum_{i=0}^{q} \sum_{Q \in \operatorname{Sing} \widetilde{\omega} \cap V_{i}} \operatorname{ind}_{Q} \widetilde{\omega}_{\mid V_{i}}
$$

for all the strata is well defined but also the sum

$$
\sum_{Q \in \operatorname{Sing} \widetilde{\omega} \cap V_{i}} \operatorname{ind}_{Q} \widetilde{\omega}_{\mid V_{i}}
$$


for each single stratum.

To prove this for a stratum $V_{i}$ we suppose that two 1-forms $\widetilde{\omega}$ and $\widetilde{\omega}^{\prime}$ have singular points of the described type. One can change each of these forms in a neighbourhood of $\overline{V_{i}} \backslash V_{i}$ by such a continuous deformation that in the course of it no new singular points arise and the value of the new forms on a fixed outward looking normal vector field on the boundary of a tubular neighbourhood of $\overline{V_{i}} \backslash V_{i}$ is positive. This can be achieved locally by adding a 1-form radial in the normal direction to a stratum (say, the differential of the squared distance from the stratum defined in local coordinates). After that, the new 1-forms $\widetilde{\omega}$ and $\widetilde{\omega}^{\prime}$, as non-vanishing sections of the cotangent bundle, are homotopic to each other in a neighbourhood of the boundary of the stratum $V_{i} \cap B_{\varepsilon}$. This implies that the sums of the indices of the singular points of the 1 -forms $\widetilde{\omega}$ and $\widetilde{\omega}^{\prime}$ inside $V_{i} \cap B_{\varepsilon}$ coincide.

Just because of the definition, the (radial) index satisfies the law of conservation of number, i.e., if a 1 -form $\omega^{\prime}$ with isolated singular points on $X$ is close to the 1 -form $\omega$, then

$$
\operatorname{ind}_{X, 0} \omega=\sum_{Q \in \operatorname{Sing} \omega^{\prime}} \operatorname{ind}_{X, Q} \omega^{\prime}
$$

where the sum on the right hand side is over all singular points $Q$ of the 1-form $\omega^{\prime}$ on $X$ in a neighbourhood of the origin. This implies the following statement. Let $X$ be a compact analytic subset of $\mathbb{R}^{N}$ with a Whitney stratification. Let $\omega$ and $\omega^{\prime}$ be 1 -forms on $\mathbb{R}^{N}$ with isolated singular points on $X$.

Proposition 1 One has

$$
\sum_{Q} \operatorname{ind}_{X, Q} \omega=\sum_{Q^{\prime}} \operatorname{ind}_{X, Q^{\prime}} \omega^{\prime}
$$

where the sums are taken over all singular points of the 1-forms $\omega$ and $\omega^{\prime}$ respectively.

Proof. This follows from the law of conservation of number applied to a family of 1-forms (with isolated singularities) connecting the 1-forms $\omega$ and $\omega^{\prime}$.

In fact one has a more precise statement:

Theorem 1 (Poincaré-Hopf) One has

$$
\sum_{Q} \operatorname{ind}_{X, Q} \omega=\chi(X)
$$

where $\chi(X)$ denotes the Euler characteristic of the set $X$.

We postpone the proof to Section 2 
Now let $(X, 0) \subset\left(\mathbb{C}^{N}, 0\right)$ be the germ of a complex analytic variety of pure dimension $n$ and let $\omega$ be a (complex and, generally speaking, continuous) 1 -form on a neighbourhood of the origin in $\mathbb{C}^{N}$. In fact there is a one-to-one correspondence between complex 1-forms on a complex manifold $M^{n}$ (say, on $\mathbb{C}^{N}$ ) and real 1 -forms on it (considered as a real $2 n$-dimensional manifold). Namely, to a complex 1-form $\omega$ one associates the real 1-form $\eta=\operatorname{Re} \omega$; the 1-form $\omega$ can be restored from $\eta$ by the formula $\omega(v)=\eta(v)-i \eta(i v)$ for $v \in T_{x} M^{n}$. This means that the index of the real 1-form $\operatorname{Re} \omega$ is an invariant of the complex 1-form $\omega$ itself. However, on a smooth manifold $\operatorname{ind}_{M^{n}, x} \operatorname{Re} \omega$ does not coincide with the usual index of the singular point $x$ of the 1 -form $\omega$, but differs from it by the coefficient $(-1)^{n}$. (E.g., the index of the (complex analytic) 1-form $\omega=\sum_{j=1}^{n} x_{j} d x_{j}\left(\left(x_{1}, \ldots, x_{n}\right)\right.$ being the coordinates of $\mathbb{C}^{n}$ ) is equal to 1 , whence the index of the real 1 -form $\operatorname{Re} \omega=\sum_{j=1}^{n} u_{j} d u_{j}-\sum_{j=1}^{n} v_{j} d v_{j}\left(x_{j}=u_{j}+i v_{j}\right)$ is equal to $(-1)^{n}$.) This explains the following definition.

Definition: The (complex radial) index $\operatorname{ind}_{X, 0}^{\mathbb{C}} \omega$ of the complex 1-form $\omega$ on $X$ at the origin is $(-1)^{n}$ times the index of the real 1-form $\operatorname{Re} \omega$ on $X$ :

$$
\operatorname{ind}_{X, 0}^{\mathbb{C}} \omega=(-1)^{n} \operatorname{ind}_{X, 0} \operatorname{Re} \omega .
$$

When it is clear from the context, the upper index $\mathbb{C}$ will be omitted.

Example. Let the variety $(X, 0)$ have an isolated singularity at the origin and let $\varepsilon>0$ be small enough so that the 1-form $\omega$ has no singular points on the intersection of $X$ with the ball $B_{\varepsilon}$ of radius $\varepsilon$ centred at the origin in $\mathbb{C}^{N}$ outside of the origin. Let $\pi:(\widetilde{X}, D) \rightarrow(X, 0)$ be a resolution of the singularity $(X, 0)$ which is an isomorphism outside of the origin. The lifting $\pi^{*} \omega$ of the 1 -form $\omega$ to the space $\widetilde{X}$ of the resolution has no zeroes outside of the exceptional divisor $D$. Let $\mathrm{Obst}_{\pi} \omega$ be the obstruction to extend the nonzero section $\pi^{*} \omega$ of the cotangent bundle of $\widetilde{X}$ from a neighbourhood of the preimage $\pi^{-1}\left(S_{\varepsilon} \cap X\right)\left(S_{\varepsilon}=\partial B_{\varepsilon}\right)$ to the whole preimage of $B_{\varepsilon} \cap X$. Since the obstruction $\mathrm{Obst}_{\pi} \omega$ (for a fixed resolution $\pi$ ) satisfies the law of conservation of number as well and for the trivial resolution of a smooth manifold $\mathrm{Obst}_{\pi} \omega$ coincides with the index $\operatorname{ind}_{X, 0} \omega$, the difference

$$
\operatorname{Obst}_{\pi} \omega-\operatorname{ind}_{X, 0} \omega
$$

does not depend on the 1 -form $\omega$. Moreover, the manifold $\widetilde{X}$ can be considered as a smoothing of the variety $X$ in the sense of [4, 5]. Therefore

$$
\operatorname{Obst}_{\pi} \omega-\operatorname{ind}_{X, 0} \omega=(-1)^{n}(\chi(D)-1)
$$

(cf. [5. Proposition 2]; note that the sign $(-1)^{n}$ appears because we consider here the complex analytic situation). 
In the sequel it will convenient to denote $\chi(Z)-1$ by $\bar{\chi}(Z)$ and to call it the reduced (modulo a point) Euler characteristic of the topological space $Z$ (though, strictly speaking, this name is only correct for a non-empty space $Z)$.

\section{The Milnor fibre of a function and the index of its differential}

We shall use the following two statements of stratified Morse theory. The first one is very general. We formulate the second one in the simplest form necessary for us. The formulations here are made in the global setting. The necessary changes for the local case (i.e. inside a ball) are obvious.

Let $X$ be a compact analytic subset of $\mathbb{R}^{N}$ with a Whitney stratification and let $f: \mathbb{R}^{N} \rightarrow \mathbb{R}$ be a smooth function with isolated critical points on $X$. For a subset $A \subset \mathbb{R}$, let $M_{A}=\{x \in X \mid f(x) \in A\}$.

Proposition 2 Suppose that the function $f$ has no critical values in the segment $[a, b]$. Then $M_{\{a\}}$ is a homotopy retract of $M_{[a, b]}$.

See the proof in 8 . It is a corollary of Thom's first isotopy lemma.

Suppose that there is only one critical point $x_{0}$ of the function $f$ on $X$ with the critical value $c$ and that in a neighbourhood of the point $x_{0}$ the function $f$ has the following form. Let $V_{i}$ be the stratum of $X$ containing $x_{0}$ and let $k$ be the dimension of $V_{i}$. Then there exists a local analytic diffeomorphism $h:\left(\mathbb{R}^{N}, \mathbb{R}^{k}, 0\right) \rightarrow\left(\mathbb{R}^{N}, V_{i}, x_{0}\right)$. Let $y_{1}, \ldots, y_{N}$ be the coordinates in $\mathbb{R}^{N}$, $\mathbb{R}^{k}=\left\{y \in \mathbb{R}^{N} \mid y_{k+1}=\ldots=y_{N}=0\right\}$. Suppose that the function $h^{*} f$ $(=f \circ h)$ is equal to the sum $f_{1}\left(y_{1}, \ldots, y_{k}\right)+f_{2}\left(y_{k+1}, \ldots, y_{N}\right)$ where the function $f_{1}:\left(\mathbb{R}^{k}, 0\right) \rightarrow(\mathbb{R}, 0)$ has a non-degenerate critical point at the origin with Morse index $m$ and $f_{2}:\left(\mathbb{R}^{N-k}, 0\right) \rightarrow(\mathbb{R}, 0)$ is an analytic germ with a strict local minimum at the origin (e.g. $\left.f_{2}\left(y_{k+1}, \ldots, y_{N}\right)=y_{k+1}^{2}+\ldots+y_{N}^{2}\right)$.

Proposition 3 For $\varepsilon>0$ small enough, the space $M_{[c-\varepsilon, c+\varepsilon]}$ can be homotopically retracted to the subspace $M_{\{c-\varepsilon\}}$ with one cell of dimension $m$ attached.

The proof is very easy. See [8] for similar, but stronger statements.

Proof of the Poincaré-Hopf theorem. Since the sum $\sum \operatorname{ind}_{X, Q} \omega$ does not depend on the 1-form $\omega$, one can take $\omega=d f$, where the smooth function $f$ has only critical points of the type described before Proposition 3 . Then Propositions 2 and 3 yield the statement.

Let $(X, 0) \subset\left(\mathbb{R}^{N}, 0\right)$ be the germ of a real analytic variety of pure dimension $n$ and let $f:(X, 0) \rightarrow(\mathbb{R}, 0)$ be the germ of an analytic function on $X$. The germ $f$ is the restriction to $X$ of the germ of an analytic function on $\left(\mathbb{R}^{N}, 0\right)$ (also denoted by $f$ ). Suppose that $f$ has an isolated critical point on 
$X$ at the origin, i.e. its differential $d f$ has an isolated singular point there. For a small enough positive $\varepsilon$, the restriction of the function $f$ to the intersection $X \cap B_{\varepsilon}$ defines a map $f: X \cap B_{\varepsilon} \rightarrow \mathbb{R}$ which is a locally trivial fibration over a punctured neighbourhood of zero in $\mathbb{R}$, generally speaking with different fibres over the positive and negative parts (see, e.g., [12]). Let $M_{f}^{+}$and $M_{f}^{-}$be the fibres of this fibration which can be called positive and negative Milnor fibres of $f$ respectively.

Theorem 2 (cf. 6, Example 2.6]) One has

$$
\operatorname{ind}_{X, 0} d f=-\bar{\chi}\left(M_{f}^{-}\right) .
$$

Proof. Let $\delta$ be positive and small enough $(0<\delta \ll \varepsilon)$ so that $M_{\{-\delta\}}=$ $f^{-1}(\delta) \cap B_{\varepsilon}$ is the negative Milnor fibre of the function $f$ and $M_{[-\delta, \delta]}=$ $f^{-1}([-\delta, \delta]) \cap B_{\varepsilon}$ is contractible. One can perturb the function $f$ in a small neighbourhood of the origin (in which the absolute value of the function $f$ is smaller than $\delta$ ) so that it will only have singular points of the type described before Proposition 3. Now the local versions of Propositions 2 and 3 yield the statement.

Let $(X, 0) \subset\left(\mathbb{C}^{N}, 0\right)$ be the germ of a complex analytic variety of pure dimension $n$ and let $f:(X, 0) \rightarrow(\mathbb{C}, 0)$ be the germ of a holomorphic function with an isolated critical point at the origin.

Theorem 3 One has

$$
\operatorname{ind}_{X, 0}^{\mathbb{C}} d f=(-1)^{n-1} \bar{\chi}\left(M_{f}\right)
$$

Proof. By [8, Proposition 2.A.3], the Milnor fibre $M_{\operatorname{Re} f}^{ \pm}$of the the real part Re $f$ of the function $f$ on $(X, 0)$ is homeomorphic to the cartesian product $M_{f} \times I$ of the Milnor fibre $M_{f}$ of the function $f$ and the segment $I=[0,1]$.

\section{The local Euler obstruction of a 1-form}

In 3], there is introduced the notion of the local Euler obstruction $\operatorname{Eu}_{f, X}(0)$ of a holomorphic function $f:(X, 0) \rightarrow(\mathbb{C}, 0)$ with an isolated critical point on the germ of a complex analytic variety $(X, 0)$. It is defined through an appropriately constructed gradient vector field tangent to the variety $X$. We adapt the definition to the case of a 1-form.

Let $(X, 0) \subset\left(\mathbb{C}^{N}, 0\right)$ be the germ of a complex analytic variety with a Whitney stratification $X=\bigcup_{i=0}^{q} V_{i}, V_{0}=\{0\}$, and let $\omega$ be a 1-form on a neighbourhood of the origin in $\mathbb{C}^{N}$ with an isolated singular point on $X$ at the origin. Let $\varepsilon>0$ be small enough such that the 1 -form $\omega$ has no singular points on $X \backslash\{0\}$ inside the ball $B_{\varepsilon}$. Let $\nu: \widehat{X} \rightarrow X$ be the Nash transformation 
of the variety $X$ defined as follows. Let $G(n, N)$ be the Grassmann manifold of $n$-dimensional vector subspaces of $\mathbb{C}^{N}$. For a suitable neighbourhood $U$ of the origin in $\mathbb{C}^{N}$, there is a natural map $\sigma: X_{\text {reg }} \cap U \rightarrow U \times G(n, N)$ which sends a point $x$ to $\left(x, T_{x} X_{\text {reg }}\right)$ ( $X_{\text {reg }}$ is the non-singular part of $\left.X\right)$. The Nash transform $\widehat{X}$ is the closure of the image $\operatorname{Im} \sigma$ of the map $\sigma$ in $U \times G(n, N)$. The Nash bundle $\widehat{T}$ over $\widehat{X}$ is a vector bundle of rank $n$ which is the pullback of the tautological bundle on the Grassmann manifold $G(n, N)$. There is a natural lifting of the Nash transformation to a bundle map from the Nash bundle $\widehat{T}$ to the restriction of the tangent bundle $T \mathbb{C}^{N}$ of $\mathbb{C}^{N}$ to $X$. This is an isomorphism of $\widehat{T}$ and $T X_{\text {reg }} \subset T \mathbb{C}^{N}$ over the regular part $X_{\text {reg }}$ of $X$. The 1-form $\omega$ gives rise to a section $\widehat{\omega}$ of the dual Nash bundle $\widehat{T}^{*}$ over the Nash transform $\widehat{X}$ without zeroes outside of the preimage of the origin.

Definition: The local Euler obstruction $\mathrm{Eu}_{X, 0} \omega$ of the 1 -form $\omega$ on $X$ at the origin is the obstruction to extend the non-zero section $\widehat{\omega}$ from the preimage of a neighbourhood of the sphere $S_{\varepsilon}=\partial B_{\varepsilon}$ to the preimage of its interior, more precisely its value (as an element of $H^{2 n}\left(\nu^{-1}\left(X \cap B_{\varepsilon}\right), \nu^{-1}\left(X \cap S_{\varepsilon}\right)\right)$ ) on the fundamental class of the pair $\left(\nu^{-1}\left(X \cap B_{\varepsilon}\right), \nu^{-1}\left(X \cap S_{\varepsilon}\right)\right)$.

The word local will usually be omitted.

Remark. The local Euler obstruction can also be defined for a real 1-form on the germ of a real analytic variety if the last one is orientable in an appropriate sense.

Example. Let $\omega=d f$ for the germ $f$ of a holomorphic function on $\left(\mathbb{C}^{N}, 0\right)$. Then $\mathrm{Eu}_{X, 0} d f$ differs from the Euler obstruction $\operatorname{Eu}_{f, X}(0)$ defined in [3] by the sign $(-1)^{n}$. The reason is that for the germ of a holomorphic function with an isolated critical point on $\left(\mathbb{C}^{n}, 0\right)$ one has $\operatorname{Eu}_{f, X}(0)=(-1)^{n} \mu_{f}$ (see 3, Remark 3.4]), whence $\operatorname{Eu}_{X, 0} d f=\mu_{f}\left(\mu_{f}\right.$ is the Milnor number of the germ $f$ ). E.g., for $f\left(x_{1}, \ldots, x_{n}\right)=x_{1}^{2}+\ldots+x_{n}^{2}$ the obstruction $\operatorname{Eu}_{f, X}(0)$ is the index of the vector field $\sum_{i=1}^{n} \bar{x}_{i} \partial / \partial x_{i}$ (which is equal to $\left.(-1)^{n}\right)$, but the obstruction $\mathrm{Eu}_{X, 0} d f$ is the index of the (holomorphic) 1-form $\sum_{i=1}^{n} x_{i} d x_{i}$ which is equal to 1 .

The Euler obstruction of a 1-form satisfies the law of conservation of number (just as the radial index). Moreover, on a smooth variety the Euler obstruction and the radial index coincide. This implies the following statement (cf. [3, Theorem 3.1]).

Proposition 4 Let $(X, 0) \subset\left(\mathbb{C}^{N}, 0\right)$ have an isolated singular point at the origin and let $\ell: \mathbb{C}^{N} \rightarrow \mathbb{C}$ be a generic linear function. Then

$$
\operatorname{ind}_{X, 0} \omega-\mathrm{Eu}_{X, 0} \omega=\operatorname{ind}_{X, 0} d \ell=(-1)^{n-1} \bar{\chi}\left(M_{\ell}\right),
$$

where $M_{\ell}$ is the Milnor fibre of the linear function $\ell$ on $X$. In particular

$$
\operatorname{Eu}_{X, 0} d f=(-1)^{n}\left(\chi\left(M_{\ell}\right)-\chi\left(M_{f}\right)\right) .
$$


Proof. One can deform the 1 -form $\omega$ to a 1 -form $\widetilde{\omega}$ such that $\ell=\widetilde{\omega}(0)$ is a generic linear function on $\mathbb{C}^{N}$. Since at the singular points of $\widetilde{\omega}$ on $X$ outside of the origin the Euler obstructions and the radial indices coincide, one has

$$
\operatorname{ind}_{X, 0} \omega-\operatorname{Eu}_{X, 0} \omega=\operatorname{ind}_{X, 0} \widetilde{\omega}-\operatorname{Eu}_{X, 0} \widetilde{\omega} .
$$

It is easy to see that both the Euler obstruction and the index of the 1-form $\widetilde{\omega}$ on $X$ at the origin coincide with those of the differential of the linear function $\ell$. The Euler obstruction $\mathrm{Eu}_{X, 0} d \ell$ is equal to zero (see [2. Lemma 1.3]) and

$$
\operatorname{ind}_{X, 0} d \ell=(-1)^{n-1} \bar{\chi}\left(M_{\ell}\right)
$$

by Theorem 3 , This yields the statement.

Now let $(X, 0) \subset\left(\mathbb{C}^{N}, 0\right)$ be an arbitrary germ of an analytic variety with a Whitney stratification $X=\bigcup_{i=0}^{q} V_{i}, V_{0}=\{0\}$. For a stratum $V_{i}, i=0, \ldots, q$, let $N_{i}$ be the normal slice in the variety $X$ to the stratum $V_{i}\left(\operatorname{dim} N_{i}=\right.$ $\left.\operatorname{dim} X-\operatorname{dim} V_{i}\right)$ at a point of the stratum $V_{i}$ and let $n_{i}$ be the index of a generic (non-vanishing) 1-form $d \ell$ on $N_{i}$ :

$$
n_{i}=(-1)^{\operatorname{dim} N_{i}-1} \bar{\chi}\left(M_{\left.\ell\right|_{N_{i}}}\right) .
$$

In particular for an open stratum $V_{i}$ of $X, N_{i}$ is a point and $n_{i}=1$. The strata $V_{i}$ of $X$ are partially ordered: $V_{i} \prec V_{j}$ (we shall write $i \prec j$ ) iff $V_{i} \subset \overline{V_{j}}$ and $V_{i} \neq V_{j} ; i \preceq j$ iff $i \prec j$ or $i=j$. For two strata $V_{i}$ and $V_{j}$ with $i \preceq j$, let $N_{i j}$ be the normal slice of the variety $\overline{V_{j}}$ to the stratum $V_{i}$ at a point of it $\left(\operatorname{dim} N_{i j}=\operatorname{dim} V_{j}-\operatorname{dim} V_{i}, N_{i i}\right.$ is a point) and let $n_{i j}$ be the index of a generic (non-vanishing) 1-form $d \ell$ on $N_{i j}: n_{i j}=(-1)^{\operatorname{dim} N_{i j}-1} \bar{\chi}\left(M_{\left.\ell\right|_{N_{i j}}}\right), n_{i i}=1$. Let us define the Euler obstruction $\mathrm{Eu}_{Y, 0} \omega$ to be equal to 1 for a zero-dimensional variety $Y$ (in particular $\mathrm{Eu}_{\overline{V_{0}}, 0} \omega=1, \mathrm{Eu}_{N_{i i}, 0} \omega=1$ ).

Theorem 4 One has

$$
\operatorname{ind}_{X, 0} \omega=\sum_{i=0}^{q} n_{i} \cdot \mathrm{Eu}_{\overline{V_{i}}, 0} \omega .
$$

Proof. There exists a 1 -form $\widetilde{\omega}$ which coincides with $\omega$ in a neighbourhood of the sphere $S_{\varepsilon}$ such that in a neighbourhood of each singular point $x_{0}$, $x_{0} \in V_{i}, \operatorname{dim} V_{i}=k$, it looks as follows. There exists a local biholomorphism $h:\left(\mathbb{C}^{N}, \mathbb{C}^{k}, 0\right) \rightarrow\left(\mathbb{C}^{N}, V_{i}, x_{0}\right)$ and, in coordinates $y_{1}, \ldots, y_{N}$ in $\mathbb{C}^{N}$ with $\mathbb{C}^{k}=\left\{y_{k+1}=\ldots=y_{N}=0\right\}$, one has $h^{*} \widetilde{\omega}=\pi_{1}^{*} \omega_{1}+d \ell$ where $\omega_{1}$ is a 1 -form on $\left(\mathbb{C}^{k}, 0\right)$ with a non-degenerate singular point at the origin (and therefore $\left.\operatorname{ind}_{\mathbb{C}^{k}, 0} \omega_{1}= \pm 1\right), \pi_{1}$ is the projection $\pi_{1}: \mathbb{C}^{N} \rightarrow \mathbb{C}^{k}$, and $\ell=\ell\left(y_{k+1}, \ldots, y_{N}\right)$ is a generic linear function on $\mathbb{C}^{N-k}$. For $i \preceq j$ one obviously has

$$
\begin{aligned}
\operatorname{ind}_{\overline{V_{j}}, x_{0}} \widetilde{\omega}=\operatorname{ind}_{\mathbb{C}^{k}, 0} \omega_{1} \cdot \operatorname{ind}_{N_{i j}, x_{0}} d \ell, \\
\operatorname{Eu}_{\overline{V_{j}}, x_{0}} \widetilde{\omega}=\operatorname{ind}_{\mathbb{C}^{k}, 0} \omega_{1} \cdot \operatorname{Eu}_{N_{i j}, x_{0}} d \ell .
\end{aligned}
$$


Since $\operatorname{Eu}_{N_{i j}, x_{0}} d \ell=0$ for $i \prec j$ [2, Lemma 1.3] and $\operatorname{Eu}_{N_{i i}, x_{0}} d \ell=\operatorname{ind}_{N_{i i}, x_{0}} d \ell=$ 1 , one has

$$
\begin{gathered}
\left.\sum_{x_{0} \in V_{j}} \operatorname{ind}_{V_{j}, x_{0}} \widetilde{\omega}\right|_{V_{j}}=\operatorname{Eu}_{\overline{V_{j}}, 0} \omega \\
\operatorname{ind}_{X, 0} \omega=\sum_{j=0}^{q} \sum_{x_{0} \in V_{j}} \operatorname{ind}_{X, x_{0}} \widetilde{\omega}=\left.\sum_{j=0}^{q} \sum_{x_{0} \in V_{j}} n_{j} \cdot \operatorname{ind}_{V_{j}, x_{0}} \widetilde{\omega}\right|_{V_{j}}=\sum_{j=0}^{q} n_{j} \cdot \operatorname{Eu}_{\overline{V_{j}, 0}} \omega .
\end{gathered}
$$

To write an "inverse" of the formula of Theorem 4 , suppose that the variety $X$ is irreducible and $X=\overline{V_{q}}$. (Otherwise one can permit $X$ to be reducible, but also permit the open stratum $V_{q}$ to be not connected and dense; this does not change anything in Theorem 4.) Let $m_{i j}$ be the (Möbius) inverse of the function $n_{i j}$ on the partially ordered set of strata, i.e.

$$
\sum_{i \preceq j \preceq k} n_{i j} m_{j k}=\delta_{i k}
$$

(see, e.g., []). For $i \prec j$ one has

$$
\begin{aligned}
m_{i j} & =\sum_{i=k_{0} \prec k_{1} \prec \ldots \prec k_{r}=j}(-1)^{r} n_{k_{0} k_{1}} n_{k_{1} k_{2}} \ldots n_{k_{r-1} k_{r}} \\
& =(-1)^{\operatorname{dim} X-\operatorname{dim} V_{i}} \sum_{i=k_{0} \prec \ldots \prec k_{r}=j} \bar{\chi}\left(M_{\left.\ell\right|_{N_{k_{0} k_{1}}}}\right) \cdot \ldots \cdot \bar{\chi}\left(M_{\left.\ell\right|_{N_{k_{r-1} k_{r}}}}\right) .
\end{aligned}
$$

Corollary 1 One has

$$
\operatorname{Eu}_{X, 0} \omega=\sum_{i=0}^{q} m_{i q} \cdot \operatorname{ind}_{\overline{V_{i}}, 0} \omega
$$

In particular

$$
\begin{aligned}
& \operatorname{Eu}_{X, 0} d f=(-1)^{\operatorname{dim} X-1} \times \\
& \qquad\left(\bar{\chi}\left(M_{\left.f\right|_{X}}\right)+\sum_{i=0}^{q-1} \bar{\chi}\left(M_{\left.f\right|_{\overline{V_{i}}}}\right) \sum_{i=k_{0} \prec \ldots \prec k_{r}=q} \bar{\chi}\left(M_{\left.\ell\right|_{N_{k_{0} k_{1}}}}\right) \ldots \bar{\chi}\left(M_{\ell_{\left.\right|_{N_{k_{r}-1} k_{r}}}}\right)\right) .
\end{aligned}
$$

\section{References}

[1] M. Aguilar, J. Seade, A. Verjovsky: Indices of vector fields and topological invariants or real analytic singularities. J. Reine Angew. Math. 504, 159176 (1998).

[2] J.-P. Brasselet, Lê D. T., J. Seade: Euler obstruction and indices of vector fields. Topology 39, 1193-1208 (2000). 
[3] J.-P. Brasselet, D. Massey, A. J. Parameswaran, J. Seade: Euler obstruction and defects of functions on singular varieties. Preprint math.AG/0302238, J. London Math. Soc. (to appear).

[4] W. Ebeling, S. M. Gusein-Zade: On the index of a vector field at an isolated singularity. In: The Arnoldfest, edited by E. Bierstone et al., Fields Inst. Commun. 24, AMS, 1999, pp. 141-152.

[5] W. Ebeling, S. M. Gusein-Zade: Indices of 1-forms on an isolated complete intersection singularity. Moscow Math. J. 3, 439-455 (2003).

[6] W. Ebeling, S. M. Gusein-Zade, J. Seade: Homological index for 1-forms and a Milnor number for isolated singularities. Preprint math.AG/0307239

[7] X. Gómez-Mont, J. Seade, A. Verjovsky: The index of a holomorphic flow with an isolated singularity. Math. Ann. 291, 737-751 (1991).

[8] M. Goresky, R. MacPherson: Stratified Morse Theory. Springer-Verlag, 1987.

[9] M. Hall: Combinatorial Theory. Second Edition. John Wiley \& Sons, New York etc., 1986.

[10] Lê D. T.: Some remarks on relative monodromy. In: Real and Complex Singularities, Oslo 1976, Sijthoff \& Noordhoff, Alphen a.d. Rijn, 1977, pp.397-403.

[11] J. A. Seade, T. Suwa: A residue formula for the index of a holomorphic flow. Math. Ann. 304, 621-634 (1996).

[12] A. N. Varchenko: Theorems on the topological equisingularity of families of algebraic varieties and families of polynomial mappings. Math. USSR Izv. 6, 949-1008 (1972).

Universität Hannover, Institut für Mathematik

Postfach 6009, D-30060 Hannover, Germany

E-mail: ebeling@math.uni-hannover.de

Moscow State University, Faculty of Mechanics and Mathematics

Moscow, 119992, Russia

E-mail: sabir@mccme.ru 